\title{
Über das Verhalten einiger Schwermetallfluoride in Lösung.
}

Von

\author{
A. JAEGER. ${ }^{1}$
}

Die Halogene Fluor, Chlor, Brom und Jod gehören der VII. Gruppe des periodischen Systems der Elemente an.

Ähnlich wie die ersten Glieder der vorhergehenden Gruppen gegenüber den anderen Gliedern derselben Gruppe grölsere Differenzen in ihrem Verhalten zeigen, unterscheidet sich auch das Fluor, trotz vieler Analogien, in hohem Mafse von den anderen Halogenen, deren Eigenschaften unter sich sehr viel ähnlicher sind und deutlich eine allmähliche Abstufung hervortreten lassen.

Diese Sonderstellung des Fluors tritt in verschiedener Weise zu Tage:

In dem Mangel einer Sauerstoffverbindung, in der Existenz zahlreicher saurer Fluoride, in den thermischen Eigenschaften der Fluorverbindungen.

Als letztere, mit deren Studium sich namentlich Morssan ${ }^{2}$ und Dewar beschäftigt haben, ist z. B. hervorzuheben, dafs das Fluor bis zu den tiefsten Temperaturen heftige Affinitätsäufserungen zeigt.

Insbesondere ist das abweichende Verhalten des Fluors auch ersichtlich aus den bei den Fluoriden auftretenden Löslichkeitserscheinungen, die vielfach im geraden Gegensatz zu denen der Chloride, Bromide und Jodide stehen.

Diese eigenartigen Löslichkeitsverhältnisse erschienen einer eingehenden Untersuchung wert und wurden daher zum Gegenstande nachstehender Arbeit gemacht, welche dazu beitragen soll, die Stellung des Fluors in der Gruppe der Halogene näher zu charakterisieren.

- Aus einer Breslauer Dissertation.

2 Morssan, Das Fluor und seine Verbindungen. Deutsche Ausgabe von Dr. Th. Zetrel, Berlin. Verlag von M. KraYN, 1900. 
Man hat gefunden, dals die verwandten Elemente sich bezüglich ihrer Löslichkeit in direkte oder umgekehrte Reihen der Atomgewichte ordnen. So nimmt in der Gruppe $\mathrm{Ba}, \mathrm{Sr}, \mathrm{Ca}, \mathrm{Mg}$ die Löslichkeit der Sulfate, Chloride und Nitrate mit steigendem Atomgewicht des Metalles ab, die der Hydroxyde zu, so zeigen die Jodide, Bromide, Chloride beim Kalium mit dem Atomgewicht abnehmende, bei den meisten edleren Metallen, z. B. $\mathrm{Pb}, \mathrm{Hg}, \mathrm{Ag}$, mit abnehmendem Atomgewicht, zunehmende Löslichkeit.

Das Fluor folgt den für die Gruppe der Halogene mafsgebenden Regeln bezüglich der Löslichkeit nicht. Es bildet z. B. nicht wịe die übrigen Halogene mit Silber unlösliche Verbindungen, dagegen bildet es solche mit den Erdalkalien, die ihrerseits mit den anderen Halogenen lösliche Verbindungen bilden.

Derartige bei den Fluorverbindungen auftretende Ausnahmeerscheinungen sollten ermittelt werden, und zwar suchte ich das Ziel zunächst durch Bestimmung der Löslichkeit von Metalloxyden in Flufssäure verschiedener Konzentration bei konstanter Temperatur zu erreichen.

Es wurde für zweckmälsig erachtet, nicht eine Lösung der Metallfuoride in Wasser vorzunehmen, sondern die Metalloxyde selbst in Flufssäure zu lösen, da man nur so die Gewifsheit hat, schliefslich eine an Oxyd gesättigte Lösung zu erhalten. Würde man die betreffenden Metallfluoride anwenden, dann würde durch hydrolytische Spaltung, d.h. unter dem Einflufs von Wasser zwar auch Flufssäure und Metalloxyd entstehen, doch wäre es möglich, daf́s letzteres nicht ausfällt, sondern etwa in ungesättigter oder in kolloidaler Lösung vorhanden wäre. Wir hätten dann das Metalloxyd nicht als Bodenkörper.

Zunächst galt es, eine Schwierigkeit zu überwinden, die allen Arbeiten mit Flufssäure sich entgegenstellt, nämlich geeignete Gefälse zur Ausführung der Untersuchungen in Anwendung zu bringen.

Die Versuche, Glasgefälse zu verwenden, die mit Paraffin oder Stearin überzogen waren, scheiterten bald an dem geringen Widerstande, welchen diese Körper der dauernden Einwirkung der Flufssäure entgegensetzten.

Die relativ grölste Widerstandsfähigkeit beobachtete ich beim reinen Bienenwachs, das wohl wegen seiner weniger krystallinischen Struktur sich dem Glase am besten anschmiegt und so der Flufssäure den Zutritt am meisten erschwert. Daher wählte ich auch 
einen Wachsüberzug für die von mir in Gebrauch genommeneri Melspipetten.

Der Überzug war hinreichend durchscheinend, so dals man die Aichmarke gut erkennen konnte.

Als Umkleidung für meine Melskolben fand ich einen passenden, recht wirksamen Stoff in einem unter dem Namen „Französischer Marineleim" in den Handel kommenden Dichtungsmittel. ${ }^{1}$

Dieser Leim schmilzt leicht zu einer mälsig dickflüssigen Masse, legt sich dann dicht an die Wandungen des Glasgefälses an und schützt dieses so vor den Angriffen der Flufssäure, Ist die Leimschicht nicht zu dick, so besteht auch hier noch genügende Transparenz zur Ablesung von Volummarken.

Zum Überziehen von Pipetten, wie überhaupt kleineren Mefsgefäfsen, eignet sich der „Französische Marineleim" kaum, weil er zuviel aufträgt. Die Löslichkeitsbestimmungen selbst wurden in dichtschliefsenden Flaschen ron ca. $80 \mathrm{ccm}$ Inhalt rorgenommen, die ich bei МЕRсK in Darmstadt aus dem altbewährten Guttapercha herstellen liefs.

So hatte ich, allerdings bei dem grofsen Nachteil, dafs die Beobachtung der Reaktion unmöglich war, auch hier die Gewähr, dafs die Flufssäure selbst bei dauerndem Gebrauch keinen Schaden thun konnte.

Bei meiner Arbeit benutzte ich Melsgefälse folgender Dimensionen:

Kolben: $200 \mathrm{ccm}, 100 \mathrm{ccm}, 50 \mathrm{ccm}$, Pipetten: $25 \mathrm{ccm}, 20 \mathrm{ccm}, 10 \mathrm{ccm}$.

Der wahre Inhalt dieser Gefälse, auf Einguls, bezw. Ausblasen bestimmt, betrug nach dem Überziehen mit der schützenden Substanz:

bezw. $180.5 \mathrm{ccm}, 95.3 \mathrm{ccm}, 48.0 \mathrm{ccm}$, bezw. $24.5 \mathrm{ccm}, 19.7 \mathrm{ccm}, 9.6 \mathrm{ccm}$.

Da meine Untersuchungen zunächst darauf gerichtet waren, Löslichkeiten von Schwermetallen in Flufssäure verschiedener Konzentration bei konstanter Temperatur zu bestimmen, stellte ich mir mit Hilfe obiger Mefsgefälse aus der als chemisch-rein käuflichen,

1 Nach einer Angabe im Chemilerkalender von 1899 kam der Marineleim erhalten werden, indem man 4 'Tage lang 1 Teil Kautschuk und 3 Teile 'Teeröl digeriert, die Flüssigkeit dekantiert und darin 3 Teile Schellack löst; mein Präparat war von der Firma Decretex \& Lejeune, Paris, bezogen. 
von mir nochmals destillierten, ca. 20 fach normalen Ausgangssäure (I) verdünnte Flufssäure von folgenden Konzentrationen her:

$\begin{array}{rrr}\text { I. HF } & \text { Titer } & 20.1 \\ \text { II. HF } & & 2.17 \\ \text { III. HF } & " & 1.11 \\ \text { IV. HE } & " & 0.57 \\ \text { V. HF } & " & 0.24 \\ \text { VI. HF } & " & 0.12\end{array}$

Nicht unerwähnt möchte ich lassen, dafs es trotz aller Vorsicht bei derartigen Arbeiten kaum möglich ist, einen leichten, durch Einatmung von Flufssäuredämpfen hervorgerufenen Katarrh zu vermeiden.

\section{Quecksilberfluorid.}

A. Nach diesen vorbereitenden Arbeiten begann ich meine Untersuchungen mit der Bestimmung der Löslichkeit ron rotem Quecksilberoxyd ${ }^{1}$ in Flufssäure, die ich in folgender Weise ausführte.

Entsprechende äquivalente Mengen $\mathrm{HgO}$ wurden in je $48 \mathrm{ccm}$ Fluissäure der verschiedenen Konzentrationen (II-VI), die sich in den Guttaperchaflaschen befanden, eingetragen. Die Versuche wurden im Schüttelthermostaten bei einer Temperatur von $25^{\circ} \mathrm{C}$. vorgenommen.

Durch Elektrolyse wurde in Proben von je $9.6 \mathrm{ccm}$ festgestellt, welche Mengen $\mathrm{HgO}$ in Lösung gegangen waren.

Die hierbei gefundenen Zahlen zeigten das Fortschreiten der Löslichkeit gemäls der Konzentration der Flufssäure und der Dauer des Versuches.

Nach längerem Schütteln ergaben sich als grölste Werte für die Löslichkeit von $\mathrm{HgO}$ in $\mathrm{HF}$, bezogen auf je $9.6 \mathrm{ccm}$ Lösungsflüssigkeit, für die verschiedenen Konzentrationen der Flulssäure folgende Zahlen:

$\begin{array}{lr}\text { HF } & \text { enthielten in } 9.6 \\ \text { Titer } & \mathrm{Hg} \text { in } \mathrm{g} \\ 0.12 & 0.0242 \\ 0.24 & 0.0475 \\ 0.57 & 0.1210 \\ 1.11 & 0.2247 \\ 2.17 & 0.4976\end{array}$

${ }^{1}$ Nach letzthin von Ostwasd ausgeführten Versuchen (s. Zeitschr, phys, Chem. 34, 495) ist die Löslichkeit des gelben Quecksilberoxyds etwas grölser, da dasselbe eine geringere Korngröfse besitzt. 
Zwischen den beiden letzten Bestimmungen bei jeder Konzentration, die durch einen verhältnismälsig gröfseren Zeitraum getrennt waren, war die Zunahme der Löslichkeit stets nur noch sehr gering, so dals man in Anbetracht der nicht gerade übermälsig weitgehenden Genauigkeit der Analysenmethode wohl die Bezeichnung .,grölste Werte" aufrecht erhalten kann. Bei monatelangem Schütteln könnte sich die Grenze der Löslichkeit vielleicht noch etwas verschieben.

Obige Zahlen habe ich in Grammatome umgerechnet und beziehe dieselben auf je $1000 \mathrm{ccm}$ Lösungsflüssigkeit.

Kurve I (s. S. 27) veranschaulicht die so erhaltenen Werte. $\mathrm{Um}$ die gefundenen Zahlen für die Löslichkeit von $\mathrm{HgO}$ in $\mathrm{HF}$ mit den theoretischen, d. h. den je $1000 \mathrm{ccm}$ der entsprechend konzentrierten Flufssäure äquivalenten Mengen $\mathrm{HgO}$ vergleichen $\mathrm{zu}$ können, habe ich die diesbezüglichen Werte in Tabelle I zusammengestellt.

Die Zahlen in der dritten Kolumne sind die der angewandten Flufssäure entsprechenden Äquivalente.

Tabelle I.

\begin{tabular}{c|c|c|c}
\hline \hline \multirow{2}{*}{$\begin{array}{c}\text { TF } \\
\text { Titer }\end{array}$} & \multicolumn{2}{|c|}{ Auf 1000 ccn Lösungsfüssigkeit g-Atome Hg } & berechnet \\
\cline { 2 - 4 } & gefunden & berechnet & gefunden \\
\hline \hline \multirow{2}{*}{0.12} & 0.01258 & 0.06 & \\
0.24 & 0.0247 & 0.12 & 4.8 \\
057 & 0.0629 & 0.285 & 4.9 \\
1.11 & 0.1168 & 0.555 & 4.5 \\
2.17 & 0.2586 & 1.085 & 4.8 \\
& & & 4.2
\end{tabular}

Wir ersehen aus Tabelle I, dafs die thatsächliche Löslichkeit von $\mathrm{HgO}$ in $\mathrm{HF}$ weit hinter der theoretisch möglichen zurückbleibt, sie beträgt, wie die Kolumne IV der Tabelle zeigt, nur ca. 20 bis $25 \%$ derselben.

Ferner geht aus der Tabelle hervor, dafs die Löslichkeit des Quecksilberoxyds in Flufssäure annähernd proportional der Konzentration der Flufssäure zunimmt; die entsprechende Kurve verläuft fast linear. Hieran knüpft sich folgende Überlegung. Die zutreffende Reaktionsgleichung sei:

$$
\mathrm{HgO}+2 \mathrm{HF} \longleftrightarrow \mathrm{HgF}_{2}+\mathrm{H}_{2} \mathrm{O}
$$




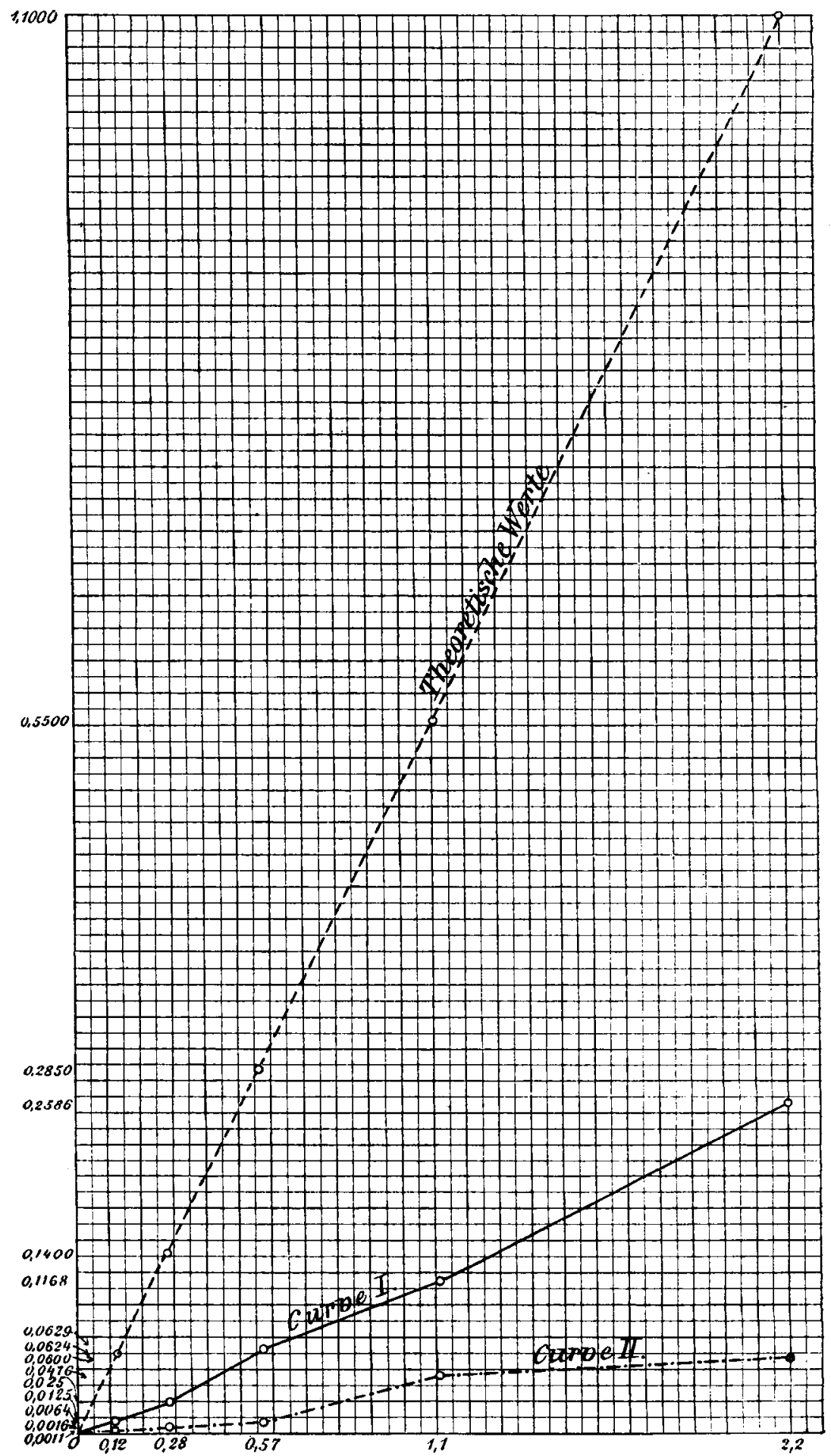


dann wäre:

$$
\left[\mathrm{HgF}_{2}\right]=\mathrm{k} \cdot[\mathrm{HF}]^{2} \text {, }
$$

d. h. die Löslichkeit mürste mit dem Quadrate der Flufssäurekonzentration zunehmen. Das gefundene Resultat dagegen führt zu der Reaktionsgleichung:

$$
\begin{gathered}
\mathrm{HgO}+\mathrm{H}_{2} \mathrm{~F}_{2} \longleftrightarrow \mathrm{HgF}_{2}+\mathrm{H}_{2} \mathrm{O} \\
{\left[\mathrm{HgF}_{2}\right]=\mathrm{k}^{\prime} \cdot\left[\mathrm{H}_{2} \mathrm{~F}_{2}\right] .}
\end{gathered}
$$

Es ist demnach durch vorstehende Versuche ein weiterer Beweisgrund erbracht worden, $\mathrm{H}_{2} \mathrm{~F}_{2}$ als die Molekularformel der Fluorwasserstoffsäure anzunehmen.

Für die Annahme einer zweimolekularen Volumzusammensetzung der Flufssäure sprechen auch die Leitfähigkeit der HF-Lösungen und nach VERnon ${ }^{1}$ der Siedepunkt des kondensierten Gases, sowie dessen zwischen $\mathrm{H}_{2} \mathrm{~F}_{2}$ und $\mathrm{HF}$ liegende Dampfdichte.

B. Es wurden nummehr Versuche angestellt, wie sich die Löslichkeit von Quecksilberoxyd in Flufssäure gestaltet, wenn man dieser vorher eine Lösung von Alkalifluorid zusetzt.

Die Wirkung solchen Zusatzes könnte entweder eine Löslichkeitserhöhung des $\mathrm{HgO}$ sein, falls sich analog, wie bei den anderen Halogenen komplexe Salze bildeten (z. B. $\mathrm{KHgF}_{3}$ ), oder es würde eine Löslichkeitsverminderung eintreten, wenn die aktive Masse der Flufssüure durch Bildung von saurem Alkalifluorid verringert wird. Das letztere ist nach meinen Versuchen der Fall.

Die Versuche wurden in folgender Weise ausgeführt: Je $24.5 \mathrm{ccm}$ Flufssäure verschiedener Konzentration wurden mit $19.7 \mathrm{ccm}$ ca. Normalfluorkaliumlösung versetzt, dazu wurden die der nunmehrigen Konzentration der Flufssäure entsprechenden Mengen Quecksilberoxyd gethan und die Flüssigkeit auf $48.0 \mathrm{ccm}$ aufgefüllt.

Die Bestimmungen wurden gleichfalls in Guttaperchaflaschen, die sich bei $25^{\circ} \mathrm{C}$. im Schüttelthermostaten befanden, vorgenommen.

Durch obige Verdünnung der Flufssäure erhielt ich zunächst die HF-Titer: $0.12,0.29,0.57,1.11$, alle aufserdem 0.41 normal an $\mathrm{KF}$.

Die gefundenen Löslichkeiten in HF mit KF-Gehalt schwanken in unregelmälsiger Weise von $0.4-0.02$ der Löslichkeiten ohne KF.

1 Nernst, Theoretisehe Chemie, 3. Aufl, S. 314. 
Da die Zahlen keine Gesetzmäfsigkeit erkemmen lassen, so sei auf ihre Wiedergabe verzichtet. Sie beweisen jedenfalls das Fehlen einer Komplexbildung, wie sie bei den anderen Halogenen bekannt ist.

\section{Kupferfluorid.}

Als zweites Metall für meine Untersuchungen wählte ich das Kupfer.

Es ist bekannt, dafs metallisches Kupfer von Flufssäure so gut wie gar nicht angegriffen wird; es lag mir daran, zu ermitteln, wie sich die Löslichkeit von $\mathrm{CuO}$ in $\mathrm{HF}$ bezw. in $\mathrm{HF}+\mathrm{KF}$ gestaltet.

Die Versuche führte ich in ganz analoger Weise wie bei $\mathrm{I}$ aus.

A. Wiederum wurden je $48 \mathrm{ccm}$ Flufssäure der verschiedenen Konzentrationen (II-VI) mit den äquivalenten Mengen $\mathrm{CuO}$ versetzt.

Die Guttaperchaflaschen wurden wiederum bei $25^{\circ} \mathrm{C}$. im Thermostaten geschüttelt.

Bei den Elektrolysen der Proben von je $9.6 \mathrm{ccm}$ Lösungsflüssigkeit ergaben sich für die verschiedenen Konzentrationen der Flufssäure nachstehende ,grölste Werte":

\begin{tabular}{|c|c|c|}
\hline $\mathrm{HF}$ & & abgeschieden $\mathrm{Cu}$ in $\mathrm{g}$ \\
\hline Titer: & & aus je $9.6 \mathrm{ccm}$ \\
\hline 0.12 & . & .0 .0187 \\
\hline 0.28 & . & . 0.0709 \\
\hline 0.57 & . & 0.1519 \\
\hline 1.08 & . & $0.2215^{1}$ \\
\hline 2.28 & . & . $0.2646^{1}$ \\
\hline
\end{tabular}

Diese Werte, ausgedrückt in Grammatomen Cu auf je $1000 \mathrm{ccm}$ Lösungsflüssigkeit, habe ich mit den "theoretischen" Werten für die Löslichkeit (d. h. der äquivalenten Mengen) von $\mathrm{CuO}$ in $\mathrm{HF}$ zu Tabelle III vereinigt.

Kurve III (s. S. 30) veranschaulicht diese Zahlen. Tabelle III.

\begin{tabular}{|c|c|c|}
\hline \multirow{2}{*}{$\begin{array}{l}\text { HF } \\
\text { Titer }\end{array}$} & \multicolumn{2}{|c|}{$\begin{array}{c}\text { Auf } 1000 \mathrm{~cm} \text { Lösungsflüssigkeit } \\
\mathrm{g} \text {-A tome Cu }\end{array}$} \\
\hline & gefunden & berechnet \\
\hline 0.12 & 0.0307 & 0.06 \\
\hline 0.28 & 0.1164 & 0.14 \\
\hline 0.57 & 0.2494 & 0.285 \\
\hline 1.08 & 0.388 & 054 \\
\hline 2.28 & 0.463 & 1.14 \\
\hline
\end{tabular}

${ }^{1}$ Die für $\mathrm{HF}_{1.08}$ und $\mathrm{HF}_{2 \cdot 28}$ angegebenen Zahlen beziehen sich auf je $9 \mathrm{ccm}$ Lösungsflüssigkeit, da für die fragliche Pipette eine Erneuerung des Überzuges notwendig geworden war. 


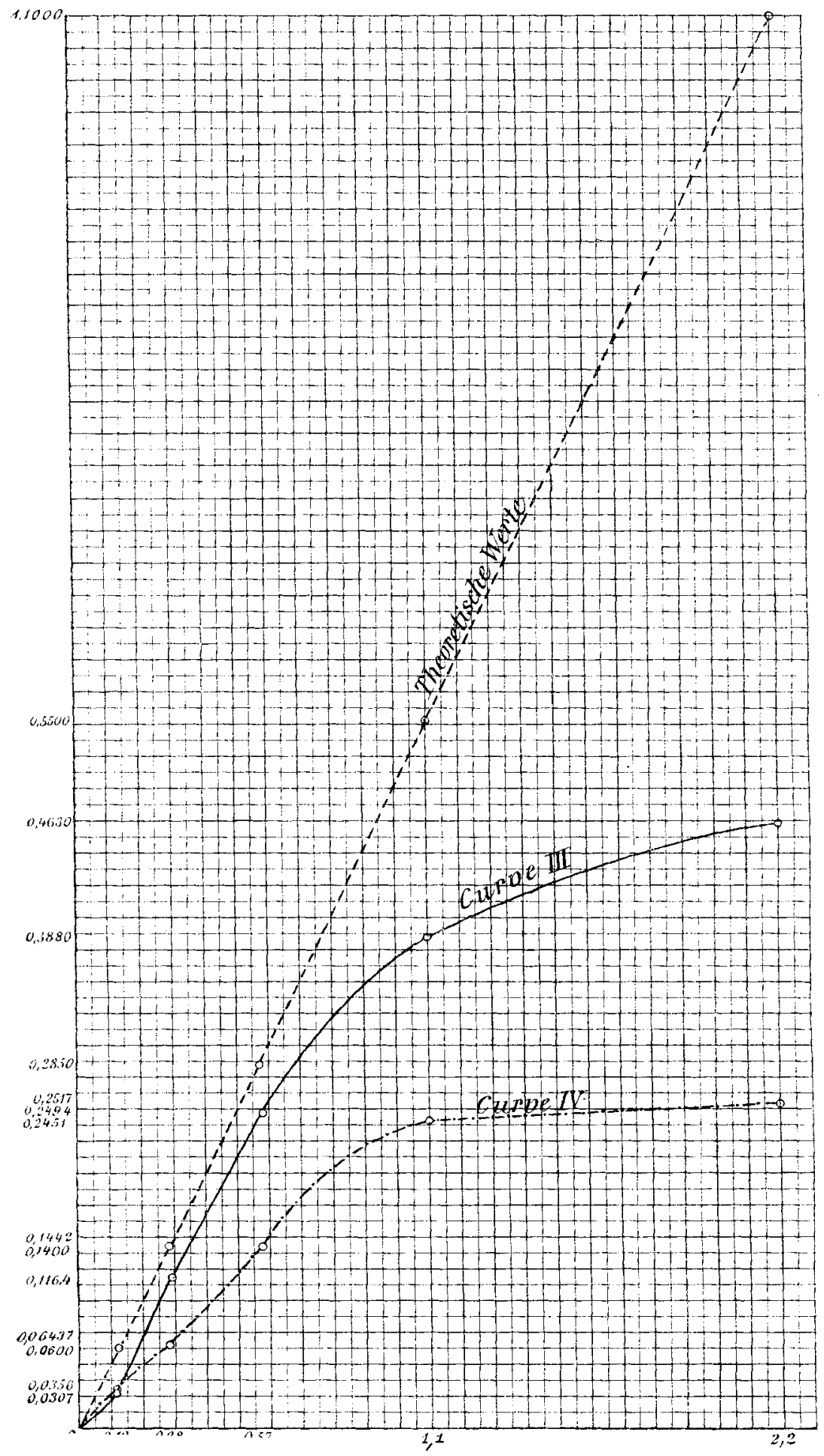


Aus Tabelle III geht zunächst hervor, dafs das Lösungsvermögen der Flufssäure dem Kupferoxyd gegenüber weit grölser ist, als gegenüber dem Quecksilberoxyd wie es auch nach den Löslichkeiten beider Oxyde ${ }^{1} \mathrm{zu}$ erwarten war.

Die für $\mathrm{HF}_{0.28}$ und $\mathrm{HF}_{0.57}$ gefundenen Zahlen kommen den theoretischen recht nahe; dagegen nimmt für die stärkeren Konzentrationen der Flufssäure die Löslichkeit nur wenig zu: Wir können hier die starke Abnahme der Dissoziation mit zunehmender Konzentration der Flufssäure beobachten.

B. Entsprechend I B habe ich auch Löslichkeitsbestimmungen für $\mathrm{CuO}$ in $\mathrm{HF}+\mathrm{KF}$ ausgeführt, bei denen ich mein Augenmerk wiederum insbesondere auf Beobachtung eventueller Komplexsalzbildungen richtete, $z u$ denen das Kupfer im allgemeinen grofse Neigung hat.

Die Versuchsanordnung war derjenigen ad I B analog.

Bei den Elektrolysen von je $9.6 \mathrm{ccm}$ der resp. Lösungen erhielt ich in dieser Versuchsgruppe folgende Werte:

$\begin{array}{cc}\mathrm{HF} & \text { abgeschieden } \mathrm{Cu} \text { in } \mathrm{g} \\ \text { Titer } & \text { auf je } 9.6 \mathrm{ccm} \text { Lösungsflüssigkeit } \\ 0.12 & 0.0217 \\ 0.28 & 0.0392 \\ 0.57 & 0.0878 \\ 1.11 & 0.1493 \\ 2.17 & 0.1533\end{array}$

Die in Kolumne II dieser Tabelle aufgeführten Zahlen ergeben Kurve IV (s. S. 30).

Tabelle IV.

\begin{tabular}{c|c|c} 
HF & $\begin{array}{c}\text { Auf } 1000 \text { cem Lösungsflüssigkeit } \\
\text { Titer }\end{array}$ & \multicolumn{2}{|c|}{ g-Atome Cu } \\
\hline & mit KF & olme KF \\
\hline 0.12 & 0.0356 & 0.0307 \\
0.28 & 0.06437 & 0.1164 \\
0.57 & 0.1442 & 0.2494 \\
$1.11(1.08)$ & 0.2451 & 0.388 \\
$2.17(2.28)$ & 0.2517 & 0.4632
\end{tabular}

Aus Tabelle IV ersehen wir, dals auch für Kupferoxyd durch Zusatz von Alkaliffuorid die Löslichkeit vermindert wird.

I Vergl. IMMERWahr, Zeitschr. Elektrochem. 7 (1901), 477. 
Nan kann demnach auch hier annehmen, dal's eine Komplexsalzbildung nicht stattfindet. Die Ausnahme für $\mathrm{HF}_{0.32}$, wo geringe Zunahme der Löslichkeit beobachtet wurde, ist wohl nur scheinbar und auf die bei dieser geringsten Konzentration der Fluissäure am stärksten hervortretenden Versuchsfehler zurückzuführen.

Leider ist eine analoge Untersuchung der Löslichkeit des Kupferoxyduls, die vielleicht eine Aufklärung der interessanten Erscheinungen gewährt hätte, nicht möglich gewesen.

Das Kupferoxydul zersetzte sich stets unter Abscheidung von metallischem Kupfer und Bildung des Oxydsalzes:

$$
\mathrm{Cu}_{2} \mathrm{O}+2 \mathrm{HF}=\mathrm{CuF}_{2}+\mathrm{H}_{2} \mathrm{O}+\mathrm{Cu} .
$$

Wenn Berzelius ${ }^{1}$ angiebt, Kupferfluorür erhalten zu haben, als er Kupferoxydul zu einem Überschufs von Fluorwasserstoffsäure fügte, und dasselbe als eine Substanz von roter Farbe, unlöslich in Wasser, Alkohol und Fluorwasserstoffsäure, beschreibt, so ist er bereits, durch Versuche von Francesco Mauro ${ }^{2}$ widerlegt worden.

Bevor ich meine Untersuchungen über das Kupferfluorid abschliefse, möchte ich noch einen Versuch erwähnen, den ich ausführte, um unmittelbar einen Mafsstab zu gewinnen für die Wirksamkeit der Flufssüure in Vergleich zu anderen Säuren.

I. Ich brachte zunächst $50 \mathrm{ccm}$ Normalessigsäure mit der äquivalenten Menge Kupferoxyd zusammen und schüttelte im Thermostaten bei $25^{\circ} \mathrm{C}$.

Bei der Elektrolyse der einzelnen Proben von je $9.6 \mathrm{ccm}$ Lösungstlüssigkeit erhielt ich hierbei abgeschiedenes Kupfer $0.1021 \mathrm{~g}$, d. h. $0.1677 \mathrm{~g}$-Atome $\mathrm{Cu}$ auf $1000 \mathrm{ccm}$ Lösungsfliassigkeit.

II. Einen analogen Versuch führte ich aus, um die Löslichkeit von Kupferoxyd in Normalsalpetersäure zu ermitteln. Die Bestimmung ergab, dafs nach melrtägigem Schütteln in $9 \mathrm{ccm}$ Lösungstlüssigkeit $0.2742 \mathrm{~g} \mathrm{Cu}$ enthalten waren. Diese Zahl entspricht $0.4802 \mathrm{~g}$-Atomen $\mathrm{Cu}$ auf $1000 \mathrm{ccm}$.

Der Theorie nach mülste man für ein zweiwertiges Metall einer normalen, einbasischen Säure gegenüber eine Löslichkeit von $0.5 \mathrm{~g}$-Atomen auf $1000 \mathrm{ccm}$ erwarten; die weitere Zunahme der

1 Pagg. Ann. 1, 28.

¿ Z. unorg. Chem. 2, 25. 
Löslichkeit bis zur Einstellung des völligen Gleichgewichtes, soweit dieselbe nicht überhaupt z. B. durch Hydrolyse beeinflufst ist, scheint nur recht langsam fortzuschreiten.

Die gefundenen Werte für die Löslichkeit des Kupferoxyds sind demnach folgende:

\section{Säure:}

$\mathrm{CH}_{3} . \mathrm{COOH}$

$\mathrm{HF}$

$\mathrm{HNO}_{3}$
g-A tome $\mathrm{Cu}$ auf $1000 \mathrm{~cm}$

Lösungsflüssigkeit

0.1677

0.388

0.4802

Vergleicht man diese für Normalessigsäure bezw. Normalsalpetersäure ermittelten Zahlen mit der für Flufssäure $\mathrm{HF}_{1^{\prime} 08}$ gefundenen, so ergiebt sich, dafs die Flufssäure in ihrer Wirksamkeit der Salpetersäure weit näher steht als etwa der Essigsäure.

Die mangelnde Gesetzmälsigkeit in den CuO-Löslichkeiten rührt wahrscheinlich von der sehr variablen Löslichkeit des Oxyds je nach seiner Modifikation her, wie auch IMmerwaHR (loc. cit.) fand.

\section{Cadmiumfluorid.}

Das Cadmiumfluorid erschien für Löslichkeitsbestimmungen besonders geeignet, weil für das Chlorid, Bromid und Jodid des Cadmiums bereits ähnliche Versuche ausgeführt worden sind und erwartet wurde, durch ergänzende Untersuchungen und Ausdehriung der sich anschlielsenden Überlegungen auf die ganze Gruppe der Halogene die hier bestehende Lücke ausfüllen zu können.

Da das Cadmiumoxyd ebenso wie das metallische Cadmium von Flufssäure nur bei Rotglut in Cadmiumfluorid übergeführt wird, mufste hier eine andere Anordnung der Versuche Platz greifen.

A) KaHLBaum'sches chemisch-reines Cadmiumfluorid wurde in Wasser eingetragen und seine Löslichkeit bei $25^{\circ} \mathrm{C}$. bestimmt.

Das Gleichgewicht suchte ich einerseits durch dauerndes Schütteln im Thermostaten zu erreichen, andererseits führte ich den Versuch auch so aus, dafs ich das Cadmiumfluorid mit Wasser erhitzte und dann die heilse Lösung bei $25^{\circ}$ stehen liefs bis zur Konstanz des Gehaltes an $\mathrm{CdF}_{2}$.

Die in Lösung gegangene Menge $\mathrm{CdF}_{2}$ wurde in beiden Fällen durch vorsichtiges Verdampfen des Lösungsmittels bestimmt.

Die gröfsten Mengen gelöstes $\mathrm{CdF}_{2}$ fand ich nach den beillen Versuchsmethoden nicht völlig übereinstimmend. 
Die Bestimmung der Löslichkeit von $\mathrm{CdF}_{2}$ in $\mathrm{H}_{2} \mathrm{O}$ durch Erhitzen, nachheriges Abkühlen auf $25^{\circ}$ und Stehenlassen, bis der Gehalt der Lösung nicht mehr abnimmt, ergab als Maximum in einer Prohe von $9 \mathrm{ccm}$ Lösungsflüssigkeit $0.3931 \mathrm{~g} \mathrm{CdF}_{2}$.

Bei der Bestimmung der Löslichkeit von $\mathrm{CdF}_{2}$ in $\mathrm{H}_{2} \mathrm{O}$ nur durch Schütteln bei $25^{\circ}$ fand ich als grölste Menge $0.4107 \mathrm{~g} \mathrm{CdF}_{2}$ in einer Probe von $9.6 \mathrm{ccm}$ Lösungsflüssigkeit.

In Mol auf $1000 \mathrm{ccm}$ Lösurgsflüssigkeit ausgedrückt, ergaben die beiden Methoden:

$$
\begin{array}{rll}
0.292 & \mathrm{Moll} \mathrm{CdF}_{2} \\
\text { bezw. } & 0.286 \mathrm{Mol} & \mathrm{CdF}_{2}
\end{array}
$$

Die geringe Differenz der beiden Resultate beruht wahrscheinlich auf Versuchsfehlern, und ich werde zum Vergleich den sich ergebenden Mittelwert heranziehen.

Was das Vergleichsmaterial betrifft, so liegen über die Löslichkeit der Halogensalze des Cadmiums neben einzelnen Angaben älterer Chemiker hauptsächlich solche von ETARD ${ }^{3}$ und in neuerer Zeit von DiEтz ${ }^{2}$ vor, welch letzterer bei seinen Bestimmungen insbesondere die verschiedenen Hydratzustände der betreffenden Salze berücksichtigt.

Das wasserfreie Cadmiumchlorid verbindet sich unter Wärmeentwickelung mit Wasser und bildet Hydrate. DiwTz giebt aufser dem Monohydrat $\mathrm{CdCl}_{2}+\mathrm{H}_{2} \mathrm{O}$ noch die beiden $\mathrm{Hydrate} \mathrm{CdCl}_{2}+21 / 2 \mathrm{H}_{2} \mathrm{O}$ und $\mathrm{CdCl}_{2}+4 \mathrm{H}_{2} \mathrm{O}$ an.

Für diese drei Hydrate hat er Löslichkeitsbestimmungen gemacht, doch führten seine Versuche, die Löslichkeit des wasserfreien Salzes zu bestimmen, zu keinem Resultat, da das Anhydrid immer unter Aufnahme von Krystallwasser in das Monohydrat überging.

Das Cadmiumbromid ist wasserfrei und als bis ca. $36^{\circ}$ beständiges Tetrahydrat bekannt. Letzteres geht oberhalb $38^{\circ}$ in $\mathrm{CdBr}_{2}+\mathrm{H}_{2} \mathrm{O}$ über.

Löslichkeitsbestimmungen sind von DIETz nur für das Monohydrat $\mathrm{CdBr}_{2}+\mathrm{H}_{2} \mathrm{O}$ und für das Tetrahydrat $\mathrm{CdBr}_{2}+4 \mathrm{H}_{2} \mathrm{O}$ ausgeführt worden.

Das Cadmiumjodid ist ebenso wie das Cadmiumfluorid nur wasserfrei bekannt. DiETz hat für das erstere Löslichkeitsbestimmungen gemacht, doch ist auch hier ein unmittelbarer Ver-

- 'Ann. Chim. Phys. 2, 503.

Z. anorg. Chem. 20, 240. 
gleich nicht angängig, da Dienz, der auch bei verschiedener Temperatur gearbeitet hat, seine Zahlen in Gewichtsprozenten der gesättigten Lösung angiebt.

ETARD bezieht alle seine Löslichkeitsbestimmungen auf wasserfreies Salz, ohne überhaupt die Existenz der Hydrate zu erwähnen, so dafs man nichts darüber erfährt, welcher Bodenkörper für die Löslichkeit anzunehmen ist.

B) Nach einer Angabe von Benzelius ist das Cadmiumfluorid in Flufssäure stärker löslich als in Wasser.

Für diese Erscheinung, die in dem Verhalten von $\mathrm{HgCl}_{2}$ zu $\mathrm{HCl}$ eine Analogie findet, war wohl ein quantitativer Beweis bisher noch nicht erbracht worden, daher machte ich neben der vorstehenden Bestimmung der Löslickkeit von Cadmiumfluorid für Wasser auch eine solche für Flufssäure.

Die Versuchsanordnung war folgende:

$48 \mathrm{ccm}$ Fluifssäure $\left(\mathrm{HF}_{1.08}\right)$, die sich in einer der erwähnten Guttaperchaflaschen befanden, wurden mit der äquivalenten Menge Cadmiumfluorid versetzt und im Thermostaten bei $25^{\circ}$ mehrere Tage geschüttelt.

Von Zeit zu Zeit wurde durch elektrolytische Abscheidung die Menge Cadmium bestimmt, welche in einer Probe von je $9 \mathrm{ccm}$ Lösungsflüssigkeit enthalten war.

Für diese Elektrolysen wurde die Form des Doppelcyanids gewählt, aus welcher sich das Cadmium als taubengrauer, festhaftender Überzug abscheidet, der sich bequem auswaschen und trocknen läfst.

Die Versuche ergaben als gröIsten Wert $0.3739 \mathrm{~g}$ abgeschiedenes Cadmium aus $9 \mathrm{ccm}$ Lösungsflüssigkeit, d. h. $0.372 \mathrm{Mol} \mathrm{CdF}_{2}$ auf $1000 \mathrm{ccm}$ Lösungsflüssigkeit.

Man hat demnach:

$$
\text { gelöst } \mathrm{CdF}_{2} \text { in } \mathrm{Mol} \text { auf }
$$

Lösungsmittel $1000 \mathrm{ccm}$ Lösungsflüssigkeit

$$
\begin{array}{cc}
\mathrm{HF}_{1,08} & 0.372 \\
\mathrm{H}_{2} \mathrm{O} & 0.289
\end{array}
$$

d. h. die Löslichkeit des Cadmiumfluorids in Flufssäure ist grölser als in Wasser. Diese Erscheinung kann man wohl nur durch Bildung eines sauren Cadmiumfluorids oder einer Komplexverbindung, Cadmiumfluorwasserstoffsäure erklären. 


\section{Bleifluorid.}

Als vierten Körper zur Untersuchung der Löslichkeit wählte ich das Bleifluorid.

Die Stellung des Bleies in der Mitte der Metalle liefs vermuten, dal's sich einer Untersuchung dieses Körpers grölsere Schwierigkeiten entgegenstellen, als einer solchen irgend eines anderen mit vorzüglicher Neigung entweder zur Komplexbildung oder zur Bildung von Einzelionen.

Es war demnach vorauszusehen, dals bei dem Blei, eigenartige, verwickelte Erscheinungen zu Tage treten werden.

A) Um die Löslichkeit von Bleioxyd in Flufssäure zu untersuchen, brachte ich, analog der vorstehend unter I und II angegebenen Methode, äquivalente Mengen $\mathrm{PbO}$ mit $\mathrm{HF}_{1 \cdot 1}$ bezw. $\mathrm{HF}_{0 \cdot 6}$ zusammen und schüttelte bei $25^{\circ}$ im Thermostaten. Nach mehrtägigem Schütteln nahm ich Proben von je $9 \mathrm{ccm}$ heraus, um die in Lösung gegangene Menge $\mathrm{PbO} z u$ bestimmen.

Es wurde die elektrolytische Bestimmung des Bleies in Form von Bleisuperoxyd gewählt.

Trotzdem ich unter Beobachtung aller Vorschriften den elektrischen Strom mehrere Stunden durch die Flüssigkeit hindurchführte, wurde keine Abscheidung von Bleisuperoxyd bemerkt; eine Gewichtszunahme der als Anode dienenden Platinschale fand nicht statt.

Dasselbe negative Resultat ergab sich auch bei meinem Versuch, Bleioxyd in circa zweifach normaler Flufssäure bezw. $\mathrm{PbO}$ in $\mathrm{HF}$ mit Zusatz von $\mathrm{KF}$ zu lösen. Ferner habe ich auch Bleioxyd mit ca. 30 fach normaler Flufssäure stark erhitzt. Die auf $25^{\circ}$ abgekühlte filtrierte Flüssigkeit enthielt, wie die Prüfung mit Schwefelammonium bezw. Kaliumbichromat ergab, ebenfalls kein Blei.

Es wäre nun zu entscheiden, ob Bleioxyd von Flufssäure überhaupt nicht angegriffen wird, ob etwa gebildetes Bleifluorid ausfällt und sich so der Untersuchung der filtrierten Lösungsflüssigkeit entzieht, oder ob es sich hier um eine Mantelbildung handelte, in der Weise, dal's zunächst gebildetes Bleifluorid eine fernere Einwirkung von Flufssäure auf das Bleioxyd verhindert.

Ich suchte zunächst das Versuchsmaterial za vergröfsern.

Metallisches Blei wird bekanntlich von Flufssäure äufserst wenig angegriffen, so dafs man häufig Flufssäure in Bleigefälsen aufbewahrt. 
Bleisuboxyd konnte zur Untersuchung nicht herangezogen werden, da auch für dieses die schon beim Kupferoxydul gemachte Beobachtung gilt, dafs es mit Säure unter Abscheidung von metallischem Blei in Bleioxyd übergeht bezw. Bleioxydverbindungen bildet.

Die höheren Oxyde des Bleies wurden wegen der bei ihnen auftretenden Zersetzungs- und Oxydationserscheinungen übergangen.

Dagegen führte ich die eingangs erwähnte Untersuchung noch für das Bleihydroxyd durch.

Bleihydroxyd stellte ich her durch Eintragen von Bleiacetatlösung in Ammoniak, eine Methode, die zuerst von TünnermanN ausgeführt wurde. Der schwere weifse Niederschlag wurde unter Absaugen filtriert, gewaschen und getrocknet. Nach DAMMer II, 2, 525 entspricht dem so hergestellten Bleihydroxyd auf Grund der gefundenen Menge Wasser die Formel 2 $\mathrm{PbO} \cdot \mathrm{H}_{2} \mathrm{O}$.

Von dem Bleihydroxyd schüttelte ich äquivalente Mengen mit $\mathrm{HF}_{2 \cdot 72}$ bezw. $\mathrm{HF}_{0.26}$ im Thermostaten bei $25^{\circ}$.

Die Untersuchung der betreffenden Lösungen ergab, dafs $9 \mathrm{ccm}$ Flulssäure vom Titer $0.26: 0.0243 \mathrm{~g} \mathrm{~Pb}$ gelöst enthielten, während in der Flulssäure vom Titer 2.72 quantitativ bestimmbare Mengen Blei nicht vorhanden waren.

$0.02425 \mathrm{~g} \mathrm{~Pb}$ auf $9 \mathrm{ccm}$ entsprechen $0.01302 \mathrm{~g}$-Atomen Blei auf $1000 \mathrm{ccm}$. Die theoretisch mögliche Löslichkeit des Bleies in $\mathrm{HF}_{0.26}$ beträgt $0.13 \mathrm{~g}$-Atome.

Der Versuch hat demnach gezeigt, dafs Bleihydroxyd wohl in schwacher Flufssäure, nicht aber in Flufssäure stärkerer Konzentration löslich ist.

Da eine Titration von $\mathrm{HF}_{2 \cdot 72}$ nach dem Versuch eine geringe Abnahme des Titers ergab, kann man wohl annehmen, dals sich hier in der That zunächst ein geringer Teil Bleifluorid gebildet hat, der in der immerhin noch sehr konzentrierten Flufssäure unlöslich ist und die weitere Menge Bleihydroxyd schützend umgiebt.

B) Behufs Ergänzung des hier gefundenen Resultates wurden weiterhin folgende Versuche ausgeführt.

1. Zunächst schüttelte ich Bleihydroxyd mit destilliertem Wasser mehrere Stunden bei Zimmertemperatur, um die eventuelle Löslichkeit zu ermitteln.

Da es schwierig ist, eine Flüssigkeit, die mit Bleihydroxyd in Berührung war, vollständig von allen Spuren Blei zu 
befreien, zeigte das Filtrat stets eine, wenn auch nur sehr geringe Trübung und schwach alkalische Reaktion; auf Zusatz von Ammoniumsulfid aber entstand ein ganz bedeutender Niederschlag von Schwefelblei.

Bei längerem Stehen der Flüssigkeit und mehrmaligem Filtrieren in ganz trockene Gefäfse erhielt ich doch schliefslich ein durchaus klares Filtrat, welches neutrale Reaktion zeigte und auf Zusatz von Schwefelammonium nicht einmal eine Trübung ergab.

Bleihydroxyd ist demnach in Wasser nicht merklich löslich; die vorher gemachten Beobachtungen der alkalischen Reaktion und des Niederschlages von Schwefelblei beruhten nur auf, dem Filtrat mechanisch beigemengten Spuren von Bleihydroxyd, das als solches alkalisch ${ }^{1}$ reagiert.

2. Durch Zusatz von Bleiacetatlösung zu den wässerigen Lösungen der betreffenden Kaliumhalogensalze stellte ich die vier Halogensalze des Bleies her.

Die Niederschläge von $\mathrm{PbCl}_{2}, \mathrm{PbBr}_{2}, \mathrm{PbJ}_{2}$ und $\mathrm{PbF}_{2}$ wurden unter Absaugen filtriert, gewaschen und getrocknet. Alle vier Salze zeigten in wässeriger Lösung schwach saure Reaktion, die durch hydrolytische Spaltung hervorgerufen wurde.

Bestimmungen der Löslichkeit von $\mathrm{PbCl}_{2}, \mathrm{PbBr}_{2}, \mathrm{PbJ}_{2}$ in $\mathrm{H}_{2} \mathrm{O}$ von v. ENDE ${ }^{2}$ ausgeführt, hatten folgendes Ergebnis:

\section{Chlorid 38.8 Millimol \\ Bromid 26.3 Millimol

Jodid $\quad 1.6$ Millimol.

Das Fluorid scheint sich nicht in diese Reihe einzufügen, denı ein Versuch ergab für die Löslichkeit desselben 5.5 Millimol auf $1000 \mathrm{ccm} \mathrm{H}_{2} \mathrm{O}$.

Wurden die vier Bleihalorde zugleich mit Bleihydroxyd in Wasser geschüttelt, dann wurde für das Chlorid Abnahme, für das Jodid und Fluorid Zunahme des Bleigehalts beobachtet, was auf Bildung basischer oder komplexer Verbindungen deutet.

3. Eine Prüfung der vier Kaliumhalogensalze hinsichtlich ihrer Reaktion auf Lackmuspapier ergab, dafs das Chlorid, Bromid und Jodid keine Färbung hervorriefen, das Fluorid rotes Lackmuspapier schwach blau, blaues schwach rot färbte. ${ }^{3}$

\footnotetext{
1 Berzelids, Pogg. Ann. 25, 396.

2 . anory. Chem. 26 (1901), 129.

3 Vergl. Aвegg und Immerwahr, Zeitschr. phys. Chem. 32, 143.
} 
Zu den Lösungen der Kaliumhalogensalze fügte ich Bleihydroxyd und schüttelte mehrere Stunden bei Zimmertemperatur. Es ergab sich hierbei folgende Änderung der Reaktion gegen Lackmus.

Das Chlorid bezw. $\mathrm{KCl}+\mathrm{Pb}(\mathrm{OH})_{2}$ reagierte schwach alkalisch, das Fluorid deutlich alkalisch, das Bromid und Jodid zeigten starke Blaufärbung des roten Lackmuspapieres.

Beim Jodid war zugleich eine beginnende Zersetzung und Jodausscheidung wahrzunehmen.

Der Nachweis von Blei jedoch in den vier Salzlösungen war weder durch Kaliumbichromat noch durch Schwefelammonium möglich. Die Änderungen der Reaktion gegen Lackmus können demnach auch nicht auf spurenhafte Beimengungen ron Bleihydroxyd zurückgeführt werden.

Den bei obigen Versuchen stattfindenden Vorgang hat man in folgender Weise darzustellen.

$$
2 \mathrm{KJ}+\mathrm{Pb}(\mathrm{OH})_{2}=2 \mathrm{KOH}+\mathrm{PbJ}_{3}
$$

dem entspricht nach dem Massenwirkungsgesetz:

oder eiufacher:

$$
\frac{\mathrm{KJ}^{2} \cdot \mathrm{h}}{\mathrm{KOH}^{2} \cdot \mathrm{j}}=\mathrm{k}
$$

$$
\frac{J^{2} \cdot h}{O H^{2} \cdot j}=k
$$

wenn $\mathrm{J}$ die Jodionenkonzentration, OH die Hydroxylkonzentration, h die Löslichkeit des Bleihydroxyd, $\mathrm{j}$ die des Bleihaloids bezeichnet.

Demnach:

oder:

$$
\mathrm{OH}=J \sqrt{\frac{\mathrm{h}}{\mathrm{j} \cdot \mathrm{k}}}
$$

$$
\begin{aligned}
& J \sqrt{\frac{\bar{h}}{\mathbf{k}}}=K \text { gesetzt. } \\
& \mathrm{OH}=\frac{\mathrm{K}}{\sqrt{\mathrm{j}}},
\end{aligned}
$$

d. h. die Hydroxylkonzentration, und damit die alkalische Reaktion ist abhängig von der spezifischen Löslichkeit des betreffenden Bleihalogensalzes; sie ist dieser umgekehrt proportional und nimmt demnach vom Chlorid nach dem Jodid hin zu, da für das Blei die Löslichkeit vom Jodid zum Bromid zum Chlorid zunimmt. 
Als Ergebnis der mitgeteilten Versuche fand sich:

Merkurifluorid ist in Lösung zu ca. $80 \%$ hydrolytisch gespalten und die Hydrolyse erfordert für die Flufssäure die Formel $\mathrm{H}_{2} \mathrm{~F}_{2}$.

Cuprifluorid ist ebenfalls hydrolysiert, doch erheblich geringer. $\mathrm{KF}$ erniedrigt die Löslichkeit von $\mathrm{HgO}$ und $\mathrm{CuO}$ in Flufssäure, wodurch die Existenz komplexer Fluoride ausgeschlossen ist.

Cadmiumfluorid ist in Wasser etwa 0.3 Molar löslich, in Fluíssäure erheblich mehr.

Bleifluorid fällt aus der Reihe der Bleihaloide durch geringe Löslichkeit heraus. Bleihydroxyd zersetzt bis zu einem Gleichgewicht mit freiem Alkali die Alkalihaloide gemäls den Bleihaloidlöslichkeiten und scheint mit den Bleihaloiden komplexe Verbindungen zu bilden.

Breslau, Chernisches Institut (phys.-chem. Abteilg.), Februar 1901.

Bei der Redaktion eingegangen am 25. Fcbruar 1901. 